\title{
Gate-controllable spin battery
}

\author{
Wen Long \\ Department of Physics, Capital Normal University, Beijing 100037, China \\ Qing-Feng Sun and Hong Guo a) \\ Center for the Physics of Materials and Department of Physics, McGill University, Montreal, PQ H3A 2T8, \\ Canada and International Center for Quantum Structures, Institute of Physics, Chinese Academy \\ of Sciences, Beijing 100080, China \\ Jian Wang \\ Department of Physics, The University of Hong Kong, Pokfulam Rood, Hong Kong, China
}

(Received 11 April 2003; accepted 20 June 2003)

\begin{abstract}
We propose a gate-controllable spin-battery for spin current. The spin battery consists of a lateral double quantum dot under a uniform magnetic field. A finite dc spin current is driven out of the device by controlling a set of gate voltages. Spin current can also be delivered in the absence of charge current. The proposed device should be realizable using present technology at low temperature. (C) 2003 American Institute of Physics. [DOI: 10.1063/1.1603331]
\end{abstract}

To be able to generate and control spin current is of great importance for spintronics. ${ }^{1}$ Traditionally, spin injection from a ferromagnetic material to a normal metal or semiconductor material has been used to obtain spin polarized charge current. Spin injection into non-Fermi liquid ${ }^{2}$ as well as by circularly polarized light $^{3}$ have also been investigated. More recently, several theoretical proposals for spin battery were reported for the generation of pure spin current without charge current. ${ }^{4-6}$ The idea is that when spin-up electrons move to one direction while an equal number of spin-down electrons move to the opposite direction, the net chargecurrent $I_{e}=e\left(I_{\uparrow}+I_{\downarrow}\right)$ vanishes and a finite spin current $I_{s}$ $=\hbar / 2\left(I_{\uparrow}-I_{\downarrow}\right)$ emerges. Here $I_{\uparrow}\left(I_{\downarrow}\right)$ is the spin-up (spindown) electron current. Although conceptually interesting, existing spin-battery proposals all involve time dependent external fields ${ }^{4-6}$ which make practical realization somewhat complicated. It is the purpose of this letter to propose and investigate a spin-battery design which is gate controllable involving no time varying fields.

The gate controllable spin battery is schematically shown in Fig. 1. It consists of a lateral double quantum-dot (QD) fabricated in two-dimensional electron gas (2DEG) with split gate technology. The two QDs are coupled to three leads: lead-1 and 3 couple to one QD each, lead-2 couples to both. The two QDs are separated by a high potential barrier so that tunnel coupling between them can be neglected. To distinguish spin of the electrons, a magnetic field $B$ is applied to the QDs to induce a Zeeman splitting. Two gate voltages $V_{g, \alpha}$ control energy levels of the $\alpha$-th $\mathrm{QD}$, where $\alpha=$ upper,lower $(u, l)$, indicating the upper and lower QD of Fig. 1. Finally, the terminal voltages for the three leads are set such that $V_{1}>V_{2}>V_{3}$ (Fig. 2), they provide energy source for the spin battery.

Before presenting results, we first discuss why the system of Fig. 1 can deliver a spin current. Due to field $B$, a spin degenerate level $\epsilon_{\alpha}$ of the $\alpha$-QD is split into spin-up/ down levels $\epsilon_{\alpha \uparrow} / \epsilon_{\alpha \downarrow}$. Let us assume $\epsilon_{\alpha \uparrow}<\epsilon_{\alpha \downarrow}$. By adjusting

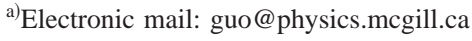

gate voltages $V_{g, \alpha}$, we shift these levels. In particular, we set $V_{g, \text { lower }}$ such that electron occupation number in the lower QD is changing between 0 and 1 (even to odd), with the level $\epsilon_{\text {lower, } \uparrow}$ locating between $\mu_{1}$ and $\mu_{2}$, where $\mu_{i}=e V_{i}$ is the chemical potential of lead $i$. Similarly, we set $V_{g \text {,upper }}$ such that the upper QD has an electron occupying state $\epsilon_{\text {upper }, \uparrow}$, while the other state $\epsilon_{\text {upper, } \downarrow}$ is pushed to higher energy $\epsilon_{\text {upper }, \downarrow}+U$ due to Coulomb interaction $U$. This way, the electron occupation number in the upper QD is changing between 1 and 2 (odd to even), and the level $\epsilon_{\text {upper, } \downarrow}+U$ locates between $\mu_{2}$ and $\mu_{3}$. The energy level diagram shown in Fig. 2 is now established. From Fig. 2, it is clear that a spin-up electron in lead-1 can tunnel into the lower QD and further to lead-2. Similarly, a spin-down electron in lead-2 can tunnel into the upper QD and flows to lead-3. Therefore, in lead-2 spin-up electrons flow in and spin-down electrons flow out: they move in opposite directions so that a net spin current is generated. Hence, by adjusting gate potentials the device of Fig. 1 generates a spin current in the region labeled by $(\mathrm{A}, \mathrm{B})$.

We now present detailed analysis. The lateral double-QD device is described by the following Hamiltonian:

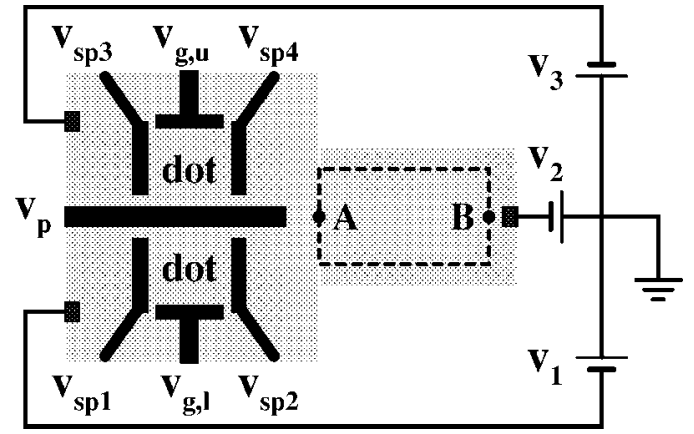

FIG. 1. Schematic diagram for the lateral quantum dot. The lightly shaded region represents two-dimensional electron gas, the darker regions are the metal gates (including split gates $V_{\mathrm{spn}}, V_{p}$, and gate voltage $V_{g, \alpha}$ ). The dotted box represents the region in which a pure spin current flows through. 


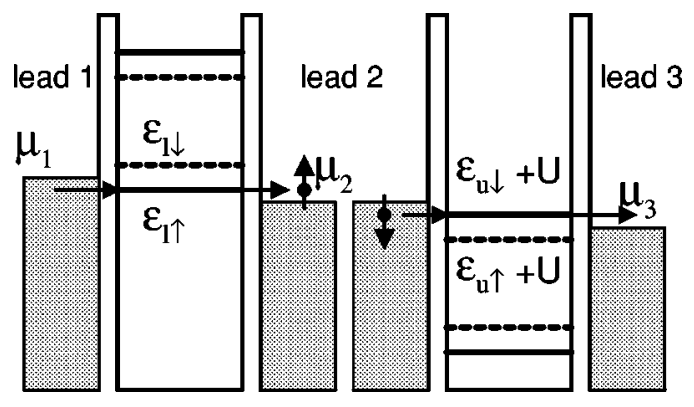

FIG. 2. Schematic plot of energy level position and the tunneling process during spin-battery operation.

$$
\begin{aligned}
H= & \sum_{\alpha \sigma}\left(\epsilon_{\alpha}-\sigma g \mu B_{\alpha} / 2\right) d_{\alpha \sigma}^{\dagger} d_{\alpha \sigma}+\sum_{\alpha} U_{\alpha} d_{\alpha \uparrow}^{\dagger} d_{\alpha \uparrow} d_{\alpha \downarrow}^{\dagger} d_{\alpha \downarrow} \\
& +\sum_{n k \sigma} \epsilon_{n k} a_{n k \sigma}^{\dagger} a_{n k \sigma}+\sum_{n k \sigma \alpha}\left(t_{n, \alpha} a_{n k \sigma}^{\dagger} d_{\alpha \sigma}+\text { H.c. }\right)
\end{aligned}
$$

where $a_{n k \sigma}^{\dagger}\left(a_{n k \sigma}\right)$ and $d_{\alpha \sigma}^{\dagger}\left(d_{\alpha \sigma}\right)$ are creation (annihilation) operators in lead- $n$ and the $\alpha$-QD, respectively. Each QD has a single particle energy level $\epsilon_{\alpha}$ with spin index $\sigma$, and the intradot Coulomb interaction is $U_{\alpha}$. To account for magnetic field $B, \epsilon_{\alpha}$ has a term $-\sigma g \mu B_{\alpha} / 2$ where $g$ is a constant. We permit $U_{\text {upper }} \neq U_{\text {lower }}$ and $B_{\text {upper }} \neq B_{\text {lower }}$, but these details do not affect our general results. The last term in the Hamiltonian describes the coupling between the QDs and the leads, and $t_{n, \alpha}$ is the coupling strength. We set $t_{1 \text {,upper }}=t_{3, \text { lower }}=0$, meaning there is no coupling between the upper-QD and lead-1 and between the lower-QD and lead-3.

We solve electron current $I_{n, \sigma}$ using standard Keldysh nonequilibrium Green's function method $(\mathrm{NEGF})^{7}(\hbar=1)$ : $I_{n \sigma}=-2 e \operatorname{Im} \Sigma_{\alpha} \int(d \epsilon / 2 \pi) \Gamma_{n \alpha}\left[f_{n}(\epsilon) G_{\alpha \sigma}^{r}(\epsilon)+\frac{1}{2} G_{\alpha \sigma}^{<}(\epsilon)\right]$ where $\Gamma_{n, \alpha} \equiv 2 \pi \Sigma_{k}\left|t_{n, \alpha}\right|^{2} \delta\left(\epsilon-\epsilon_{n k}\right)$ is the linewidth function. $f_{n}(\epsilon)$ is the Fermi distribution function in lead- $n$. The NEGF $G_{\alpha \sigma}^{r,<}(\epsilon)$ is the Fourier transform of $G_{\alpha \sigma}^{r,<}(t)$ : with $G_{\alpha \sigma}^{r}(t) \equiv-i \theta(t)\left\langle\left\{d_{\alpha \sigma}(t), d_{\alpha \sigma}^{\dagger}(0)\right\}\right\rangle \quad$ and $\quad G_{\alpha \sigma}^{<}(t)$ $\equiv i\left\langle d_{\alpha \sigma}^{\dagger}(0) d_{\alpha \sigma}(t)\right\rangle$.

We solve the retarded Green's function $G_{\alpha \sigma}^{r}$ in by the standard equation of motion technique where indirect tunneling processes such as upper QD $\rightarrow$ lead- $\rightarrow$ lower QD are neglected, this is reasonable because the long middle barrier between the QDs helps to block such events to a large extent. We obtain 6

$$
G_{\alpha \sigma}^{r}(\epsilon)=\frac{\epsilon_{\alpha \sigma}^{-}+U_{\alpha} n_{\alpha \bar{\sigma}}}{\left(\epsilon-\epsilon_{\alpha \sigma}\right) \epsilon_{\alpha \sigma}^{-}+\frac{i}{2} \Gamma_{\alpha}\left(\epsilon_{\alpha \sigma}^{-}+U_{\alpha} n_{\alpha \bar{\sigma}}\right)},
$$

where $\quad \epsilon_{\alpha \sigma}^{-} \equiv \epsilon-\epsilon_{\alpha \sigma}-U_{\alpha}, \quad \epsilon_{\alpha \sigma} \equiv \epsilon_{\alpha}-\sigma g \mu B_{\alpha} / 2, \quad \Gamma_{\alpha}$ $=\Sigma_{n} \Gamma_{n \alpha}$, and $n_{\alpha \bar{\sigma}}$ is the intradot occupation number of state $\bar{\sigma}$ in the $\alpha$-QD. $n_{\alpha \bar{\sigma}}$ needs to be calculated self-consistently from the self-consistent equation $n_{\alpha \bar{\sigma}}=$ $-i \int(d \epsilon / 2 \pi) G_{\alpha \sigma}^{<}(\epsilon)$. As usual, $G_{n \sigma}^{r}(\epsilon)$ has two resonances: one at energy $\epsilon_{\alpha \sigma}$ for which the associated state $\epsilon_{\alpha \bar{\sigma}}$ is empty; the other is at $\epsilon_{\alpha \sigma}+U_{\alpha}$ for which the associated state $\epsilon_{\alpha \bar{\sigma}}$ is occupied.

Following the approach of Ref. 8, we obtain $\int d \epsilon G_{\alpha \sigma}^{<}(\epsilon)$ which is needed in computing current and occupation number Would depends on the field direction. (iii) The four coupling
Downloaded 07 Nov 2006 to 147.8.21.97. Redistribution subject to AIP license or copyright, see http://apl.aip.org/apl/copyright.jsp

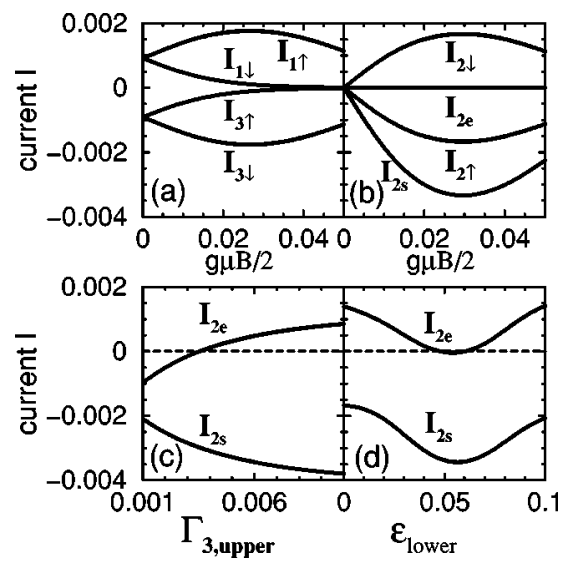

FIG. 3. (a) and (b) for electron currents $I_{n \uparrow}$ and $I_{n \downarrow}$, charge current $I_{2 e}$ (unit $e$ ), and spin current $I_{2 s}$ (unit $\hbar / 2$ ), vs magnetic field parameter $g \mu B / 2$. Other parameters are $\Gamma_{1, \text { lower }}=\Gamma_{2, \text { lower }}=\Gamma_{2 \text {,upper }}=\Gamma_{3, \text { upper }}=0.005, \quad k_{B} T$ $=0.01, U_{\text {lower }}=1.0, U_{\text {upper }}=0.9, \epsilon_{\text {lower }}=\mu_{1}$, and $\epsilon_{\text {upper }}+U_{\text {upper }}=\mu_{3}$. (c) $I_{2 e}$ and $I_{2 s}$ vs $\Gamma_{3 \text {,upper }}$ with $g \mu B / 2=0.03$. Other parameters are: $\Gamma_{1, \text { lower }}$ $=0.004, \Gamma_{2, \text { lower }}=0.005$, and $\Gamma_{2 \text {,upper }}=0.006$. (d) $I_{2 e}$ and $I_{2 s}$ vs $\epsilon_{\text {lower }}$ with $g \mu B / 2=0.03$. Here $\epsilon_{\text {upper }}+U_{\text {upper }}=-0.06$ which is slightly different from $\mu_{3}=-0.05$. Other parameters in (c) and (d) are the same as those in (a) and (b)

$\int \frac{d \epsilon}{2 \pi} G_{\alpha \sigma}^{<}(\epsilon)=-\int \frac{d \epsilon}{2 \pi} \sum_{n} \frac{\Gamma_{n \alpha} f_{n}}{\Gamma_{\alpha}}\left[G_{\alpha \sigma}^{r}(\epsilon)-G_{\alpha \sigma}^{a}(\epsilon)\right]$.

This completes the analytical derivation.

We set bias voltages $\mu_{1}=0.05, \mu_{2}=0, \mu_{3}=-0.05$ so that $\mu_{1}>\mu_{2}>\mu_{3}$. We set gate voltages $V_{g, \alpha}$ such that at zero magnetic field, $\epsilon_{\text {lower }}=\mu_{1}$ and $\epsilon_{\text {upper }}+U_{\text {upper }}=\mu_{3}$. With this condition there is one electron in the upper QD. Figures 3(a) and 3(b) shows electron current $I_{n \uparrow}$ and $I_{n \downarrow}$; charge current in lead-2 $I_{2 e}=e\left(I_{2 \uparrow}+I_{2 \downarrow}\right)$; and spin current in lead-2 $I_{2 s}=(\hbar / 2)\left(I_{2 \uparrow}-I_{2 \downarrow}\right)$, versus a uniform field strength $B$. At zero $B$, electron current is nonpolarized so that $I_{n \uparrow}=I_{n \downarrow}$, and both $I_{2 e}$ and $I_{2 s}$ vanish. When $B$ increases from zero, the intradot level $\epsilon_{\alpha}$ is split. Then levels $\epsilon_{\text {lower, } \uparrow}$ and $\epsilon_{\text {upper, } \downarrow}$ $+U_{\text {upper }}$ are moved into the bias "window" between $\mu_{1}\left(\mu_{3}\right)$ and $\mu_{2}$, while levels $\epsilon_{\text {lower, } \downarrow}$ and $\epsilon_{\text {upper, } \uparrow}+U_{\text {upper }}$ are moved out of the window, see Fig. 2. In this situation the electron current in lead-1 and lead-3 are polarized with $I_{\alpha \uparrow} \neq I_{\alpha \downarrow}$. Moreover, we have $\left|I_{1 \uparrow}\right|>\left|I_{1 \downarrow}\right|$ and $\left|I_{3 \uparrow}\right|<\left|I_{3 \downarrow}\right|$. In the following, we focus on current in lead-2, shown in Fig. 3(b). In lead-2 the value of electron current $I_{2 \uparrow}$ equals to the value of $I_{2 \downarrow}$, but their flow direction is exactly opposite to each other, hence, we have $I_{2 \uparrow}=-I_{2 \downarrow}$. We therefore obtain zero charge current $I_{2 e}=0$; and a net spin current $I_{2 s}$ emerges. When parameter $g \mu B / 2 \approx 0.03$, the indradot levels $\epsilon_{\text {lower, } \uparrow}$ and $\epsilon_{\text {upper, } \downarrow}+U_{\text {upper }}$ are in the middle of the bias window, leading to the maximum spin current. If field $B$ increases further, the spin current slightly decreases.

The device discussed here should be realizable using present technology because lateral double-QD structures have already been fabricated. ${ }^{9}$ Our analysis also show that the device does not have a very strict parameter requirement. (i) The sizes of the two QDs need not be the same; the intradot Coulomb interaction parameters $U_{\text {upper }}, U_{\text {lower }}$ need not be the same. (ii) The field $B$ may or may not be uniform, it may also point to any direction. For different directions of B, a spin current is still induced but the spin polarization 
strengths $\left(\Gamma_{1, \text { lower }}, \Gamma_{2, \text { lower }}, \Gamma_{3 \text {,upper }}, \Gamma_{2 \text {,upper }}\right)$ between the QDs and the leads can be controlled by split gate voltages $\left(V_{\mathrm{sp} 1}, V_{\mathrm{sp} 2}, V_{\mathrm{sp} 3}, V_{\mathrm{sp} 4}\right)$ as shown in Fig. 1, and they do not need to be the same. In fact, one may fix any 3 of the 4 and only regulate the last one to obtain a pure spin current with zero charge current. For example, fixing $\Gamma_{1, \text { lower }} \neq \Gamma_{2 \text {,lower }}$ $\neq \Gamma_{2, \text { upper }}$, the spin current $I_{2 s}$ and charge current $I_{2 e}$ vs $\Gamma_{3 \text {,upper }}$ is shown in Fig. 3(c). At a special value of $\Gamma_{3 \text {,upper }}$ given by relation $\Gamma_{3 \text {,upper }} \Gamma_{2 \text {,upper }} / \Gamma_{\text {upper }}$ $=\Gamma_{1, \text { lower }} \Gamma_{2, \text { lower }} / \Gamma_{\text {lower }}, I_{2 e}$ vanishes and only $I_{2 s}$ exists. (iv) So far we have set $\epsilon_{\text {lower }}=\mu_{1}$ and $\epsilon_{\text {upper }}+U_{\text {upper }}=\mu_{3}$, but these conditions can be relaxed. For example, if $\epsilon_{\text {upper }}$ $+U_{\text {upper }}=-0.06$, somewhat different from $\mu_{3}$, by regulating the lower-QD level $\epsilon_{\text {lower }}$ using gate voltage $V_{g \text {,lower }}$, we can easily find the operation point for large $I_{2 s}$ with zero $I_{2 e}$, as shown in Fig. 3(d). (v) As for the parameter values, in Fig. 3 we have used $k_{B} T=0.01$. Assuming this is equivalent to $100 \mathrm{mK},{ }^{10}$ other parameter values used to generate Fig. 3 can be deduced. We find: $V_{1}=\mu_{1} / e \approx 43 \mu \mathrm{V}, V_{2}=0, V_{3} \approx$ $-43 \mu \mathrm{V}, U_{\alpha} \approx 1 \mathrm{meV}$, and $B \approx 0.8 / \mathrm{g} \mathrm{T}$ for $g \mu B / 2=0.03 .{ }^{11}$ These parameters are in the standard range of QD devices. ${ }^{12}$

Finally, we discuss in what sense the proposed device behaves as a spin battery with two poles. Note that the region indicated by the dotted box in Fig. 1 is reserved for spintronic devices: any application of spin current should be done in this region. The lateral QD plus the external circuit constitute the spin battery: the two poles of the spin battery are points "A" and "B" as shown in Fig. 1. If there exists direct connection between $\mathrm{A}$ and $\mathrm{B}$, a spin current is driven through by the spin battery. On the other hand, if there is no direct connection, a spin-motive force will be established between A and B. Importantly, even if there are not spin flip mechanisms in whole device, the spin battery can still work, which is different from the one-pole systems. ${ }^{4,5}$ Finally, the distance between points A and B can be as large as the spin coherence length which can reach many microns at low temperatures. ${ }^{13,14}$ Such as large distance should allow useful applications of the flowing spin current.
In summary, we have shown that gate-controllable spin battery for spin current is possible. Such a device should be fabricable using present technology. We believe the present design to be superior as no time-dependent field is involved. In the present work, we did not discuss detection of pure spin current without charge current, but such discussions already exist in literature ${ }^{13,15,16}$ and we refer interested readers to them.

The authors gratefully acknowledge financial support from NSERC of Canada, FCAR of Quebec (Q.S., H.G), the National Science Foundation of China, the Chinese Academy of Sciences (Q.S.), and a RGC grant from the SAR Government of Hong Kong under Grant No. HKU 7091/01P (J.W.).

${ }^{1}$ S. A. Wolf, D. D. Awschalom, R. A. Buhrman, J. M. Daughton, S. V. Molnar, M. L. Roukes, A. Y. Chtchelkanova, and D. M. Treger, Science 294, 1488 (2001); G. A. Prinz, ibid. 282, 1660 (1998).

${ }^{2}$ Q. Si, Phys. Rev. Lett. 81, 3191 (1998).

${ }^{3}$ I. Zutic, J. Fabian, and S. D. Sarma, Appl. Phys. Lett. 79, 1558 (2001); 82, 221 (2003).

${ }^{4}$ A. Brataas, Y. Tserkovnyak, G. E. W. Bauer, and B. Halperin, Phys. Rev. B 66, 060404 (2002).

${ }^{5}$ B. Wang, J. Wang, and H. Guo, Phys. Rev. B 67, 092408 (2003).

${ }^{6}$ Q.-F. Sun, H. Guo, and J. Wang, Phys. Rev. Lett. 90, 25830 (2003).

${ }^{7}$ Y. Meir and N. S. Wingreen, Phys. Rev. Lett. 68, 2512 (1992).

${ }^{8}$ Q.-F. Sun and H. Guo, Phys. Rev. B 66, 155308 (2002).

${ }^{9}$ M. Ciorga, A. S. Sachrajda, P. Hawrylak, C. Gould, P. Zawadzki, S. Jullian, Y. Feng, and Z. Wasilewski, Phys. Rev. B 61, R16315 (2000).

${ }^{10}$ If tempearture is too high, e.g., $k_{B} T>5 g \mu B$, spin current will be diminished due to thermal fluctuations.

${ }^{11}$ For typical GaAs/AlGaAs $2 \mathrm{DEG}, g \sim 0.45$ so that $B \approx 2 \mathrm{~T}$.

${ }^{12}$ In our model Hamiltonian, we consider only one level for each QD. This is reasonable if the level spacing $\Delta$ is larger than the bias $\left|V_{1 / 3}-V_{2}\right|$. This should be realizable in present technology. For example, for a $0.2 \mu \mathrm{m}$ $\times 0.2 \mu \mathrm{m}$ QD, its $\Delta$ is about $\pi \hbar /\left(m^{*}\right.$ area $) \approx 90 \mu \mathrm{V}>\left|V_{1 / 3}-V_{2}\right|$ $\approx 43 \mu \mathrm{V}$.

${ }^{13}$ S. Zhang, J. Appl. Phys. 89, 7564 (2001).

${ }^{14}$ M. Johnson and R. H. Silsbee, Phys. Rev. Lett. 55, 1790 (1985); Phys. Rev. B 37, 5312 (1988).

${ }^{15}$ B. Heinrich, G. Woltersdorf, R. Urban, and E. Simanek, J. Magn. Magn. Mater. 258, 376 (2003).

${ }^{16}$ J. E. Hirsh, Phys. Rev. Lett. 83, 1834 (1999). 